\title{
MINI-REVIEW
}

\section{Cannabis and the Gut-Brain Axis Communication in HIV Infection}

\author{
Natalie L. Wilson, ${ }^{1 * *}$ Scott N. Peterson, ${ }^{2}$ and Ronald J. Ellis ${ }^{3}$
}

\begin{abstract}
People living with HIV infection (PWH) disclose that cannabis is an effective strategy for alleviating symptoms associated with HIV disease. However, some medical providers feel ill-informed to engage in evidence-based conversations. HIV leads to alterations in the gut microbiome, gut-brain axis signaling, and chronic inflammation. The endocannabinoid system regulates homeostasis of multiple organ systems. When deficient, dysregulation of the gut-brain axis can result in chronic inflammation and neuroinflammation. Cannabis along with the naturally occurring endocannabinoids has antioxidant and anti-inflammatory properties that can support healing and restoration as an adjunctive therapy. The purpose of this literature review is to report the physiologic mechanisms that occur in the pathology of HIV and discuss potential benefits of cannabinoids in supporting health and reducing the negative effects of comorbidities in PWH.
\end{abstract}

Keywords: endocannabinoids; cannabis; gut-brain axis; HIV; microbiome

\section{HIV and Cannabis}

People living with HIV infection (PWH) often search for ways to manage symptoms to improve their quality of life. Cannabis is an effective strategy for alleviating symptoms associated with HIV disease and with other conditions such as cancer (i.e., nausea, sleep disorders, musculoskeletal and neuropathic pain, anxiety, and depression). ${ }^{1-7}$ Indeed, several meta-analyses of clinical trials have established the effectiveness of cannabis for HIV-related neuropathic pain and nausea, ${ }^{1,8}$ although dosing and administration routes have varied widely. Some studies suggest that titrating dosing to effectiveness and side effects is a useful strategy for dose selection. ${ }^{5}$

PWH disclose, to their health care providers and in unpublished qualitative interviews (N.L. Wilson, C. Ott, unpublished data), using community-acquired cannabis to self-manage symptoms such as poor appetite, gastrointestinal (GI) and sleep disorders, pain, or to improve adherence to antiretroviral therapy (ART) food intake requirements by increasing appetite (N.L. Wilson, C. Ott, unpublished data). ${ }^{9,10}$ However, some medical providers feel ill-informed to engage the patient in evidence-based conversations regarding cannabis selection, beneficial effects, adverse effects, and harm reduction. ${ }^{10,11}$ Yet, due to federal restrictions, it is difficult to run clinical trials to address better informed evidence-based conversations.

Medicinal cannabis is rapidly becoming an acceptable and state-level authorized form of symptom and disease management. ${ }^{12,13}$ Health care providers understand the potential benefits of cannabis for symptom management. However, clinicians feel hindered by a lack of information to make recommendations and have an informed discussion of risk reduction, potential dependence, or possible adverse effects with their patients currently using or desiring to use cannabis. ${ }^{10,11}$ Among 71 clinicians that had authorized medicinal cannabis for their patients in Washington State, $56.4 \%$ had limited knowledge of available products and where to get them, and $70 \%$ were uncertain of dosing. ${ }^{11}$ An improved understanding of the strategic use of cannabis would support clinicians in their discussions with patients. Compounding this, research on

\footnotetext{
${ }^{1}$ Department of Community Health Systems, School of Nursing, University of California, San Francisco, San Francisco, California, USA.

${ }^{2}$ Sanford Burnham Prebys Medical Discovery Institute, La Jolla, California, USA.

${ }^{3}$ Departments of Neurosciences and Psychiatry, University of California, San Diego, San Diego, California, USA.

*Address correspondence to: Natalie L. Wilson, PhD, DNP, MPH, APRN-BC, University of California, San Francisco, School of Nursing, 2 Koret Way, N531B, San Francisco, CA 94143, USA, E-mail: natalie.wilson@ucsf.edu
} 
the effects of cannabis in PWH are generally lacking and as such fail to provide clinicians with reliable data for cannabis use recommendations.

Cells throughout the human body express a network of receptors and enzymes referred to as the endocannabinoid system for the synthesis and sensing of endogenous lipid ligands. The overarching function of the endocannabinoid system is to mediate regulation of energy utilization and substrate metabolism to maintain a relative state of equilibrium or balance in the body's physiological and interdependent processes, for example, homeostasis. ${ }^{14-16}$ Endocannabinoid system signaling pathways have been pursued as a target for future pharmacotherapy to reduce inflammation and provide therapy in pathological conditions. ${ }^{15,17}$ Components of cannabis bind to endocannabinoid receptors, modulating the function of the endocannabinoid system.

The endocannabinoid system is particularly important in HIV infection for a number of reasons. For example, cannabis use is associated with a reduction in systemic inflammation and immune activation, ${ }^{18}$ and there is a more rapid decline of HIV DNA among cannabis users taking ART than with those not using cannabis. ${ }^{19}$ Reductions in inflammation and more rapid declines in HIV DNA might contribute to lower viral loads and higher CD4 T cells in PWH cannabis users. $^{20,21}$ We recently reported that more recent use was associated with significantly lower Interleukin (IL)-16 levels in cerebrospinal fluid (CSF) and lower soluble tumor necrosis factor (TNF) receptor type-II and inducible protein (IP)-10 levels in plasma. ${ }^{22}$

This review will outline the endocannabinoid system and phytocannabinoids (e.g., cannabis) and their effect on the gut-brain axis (GBA) in the context of HIV infection.

\section{HIV and the Gut}

HIV infection is associated with systemic $\mathrm{CD} 4^{+} \mathrm{T}$ cell depletion, gut dysbiosis, gut epithelial barrier dysfunction, chronic inflammation, and consequential poor health outcomes, ${ }^{23-26}$ even when well controlled by antiretroviral therapy. ${ }^{27}$ Gut barrier dysfunction permits the translocation of inflammatory microbial products such as a lipopolysaccharide (LPS; a cell wall component). ${ }^{23,25,27}$ In particular, following viral control with $\mathrm{ART}, \mathrm{CD} 4^{+} \mathrm{T}$ cells do not return to normal levels in the gut, and gut epithelium defects are highly correlated with this persistent gut $\mathrm{CD} 4{ }^{+}$cell depletion. Increased apoptosis, chronic inflammatory signals, and reduced proliferation and repair of epithelial cells all contribute to compromised gut barrier function. Altered tryptophan metabolism and deficits in butyrate producing microbes in PWH have been reported and likely also contribute to increased gut permeability. ${ }^{28-30}$ Barrier defects render $\mathrm{HIV}^{+}$individuals susceptible to increased local and systemic exposure to pro-inflammatory ligands produced by gut microbiota. ${ }^{31-33}$ Together these alterations lead to poor HIV disease outcomes, including HIV-associated neurocognitive disorders (HAND). ${ }^{24}$ In this article, we present possible physiologic influence of cannabis on gut barrier integrity in PWH (Fig. 1).

\section{Gut Microbiome and HIV}

The gut microbiota regulates and maintains functional activities of the gut mucosal barrier integrity, the gut associated lymphoid tissue (GALT), immunomodulation, and protection against pathogenic organisms. ${ }^{34}$ The diverse bacterial community comprising the gut microbiota contains both beneficial and detrimental microorganisms. ${ }^{35}$ Beneficial species interact with the host immune system in a mutualistic manner. ${ }^{36}$ One of the main functions of the gut microbiota is to communicate with and regulate immune functions that impact immune cells in close proximity, for example, GALT, residing basolaterally to the monolayer of gut epithelium, ${ }^{37}$ as well as distal immune responses. The GALT represents $70-80 \%$ of the cellular immune compartment and is the largest immune reservoir in the human body. Early in infection, HIV establishes a high titer in the GALT. Ongoing HIV replication is responsible for the depletion of GALT CD4 ${ }^{+} \mathrm{T}$ cells and subsequent alterations of the gut microbiota. ${ }^{38,39}$ Even in the context of antiretroviral treatment and virologic suppression, patients continue to show increased inflammation and immune cell activation, as well as elevated gut permeability and dysbiotic microbiota. ${ }^{24,40}$ While normal commensal flora contribute to tolerance and balance between $\mathrm{T}$ helper subset pattern recognition receptor signaling, ${ }^{37}$ loss or replacement of these beneficial flora leads to loss of T-helper cell type 1 (TH1) function, amplifying GALT dysfunction in HIV infection. Depletion of Th17 cells in the GALT leads to reduced IL-22 production diminishing epithelium repair processes and maintenance of tight gap junctions. Such barrier defects create a pathway for microbial products to escape the gut lumen and enter the systemic circulation. The entry of microbial products into the blood triggers the innate immune response and release of pro-inflammatory cytokines, such as IL- $1 \beta$, TNF- $\alpha$, and others ${ }^{26,27}$ (Fig. 1). 


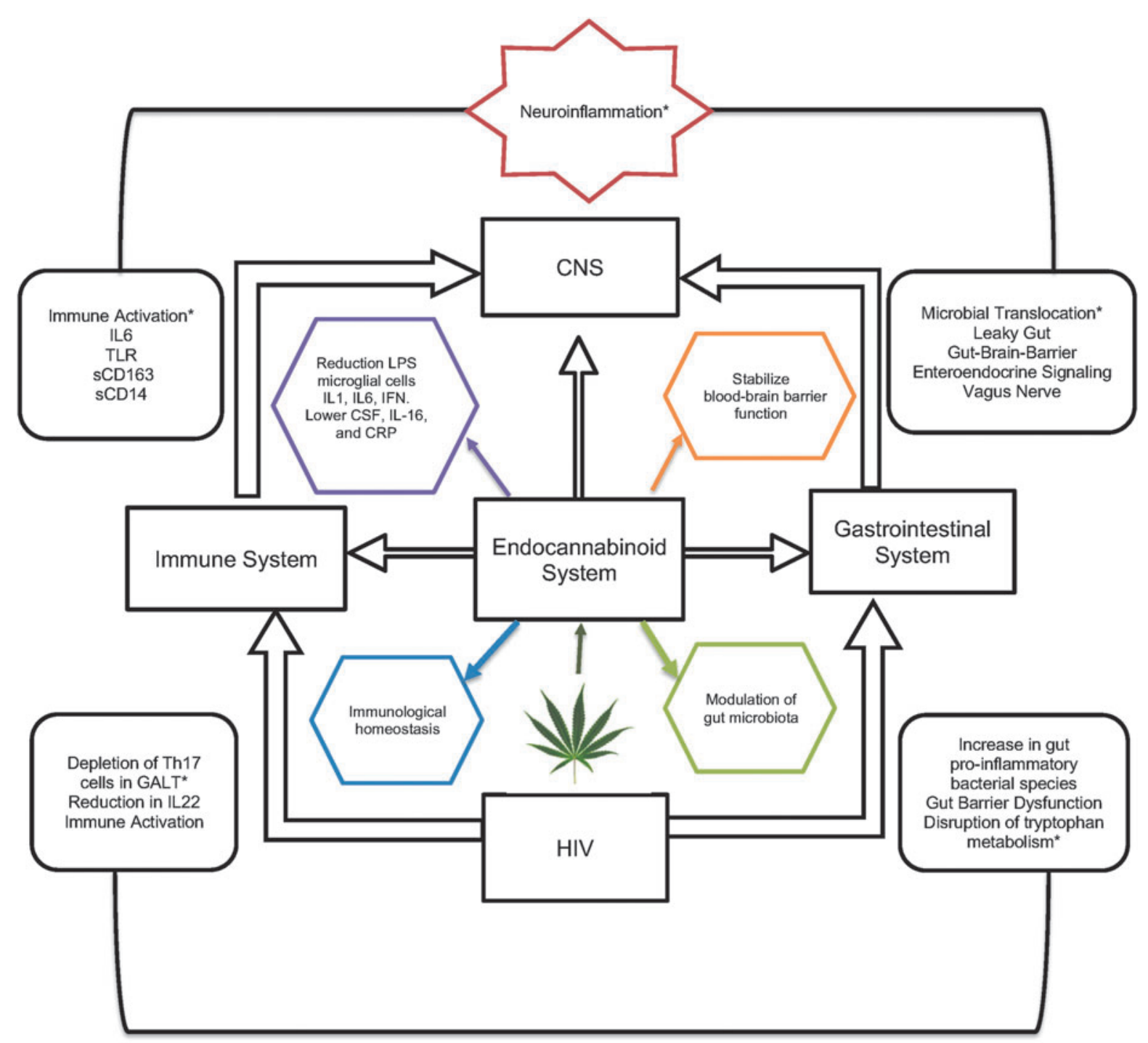

FIG. 1. The effects of endocannabinoids and phytocannabinoids in HIV infection. HIV has a detrimental effect on the immune and gastrointestinal systems leading to immune activation and chronic inflammation of the CNS. *Abnormalities persist despite ART. The homeostasis role of endocannabinoid system provides a mechanism for temporary restoration of balance within these systems. Cannabis has anti-inflammatory and antioxidant properties, which serve in the place of endocannabinoids when deficient. ART, antiretroviral therapy; CNS, central nervous system; CSF, cerebrospinal fluid; GALT, gut associated lymphoid tissue.

Microbial dysbiosis is associated with numerous adverse effects, including impaired function of the gut barrier and translocation of microbial products into the bloodstream causing systemic inflammation. ${ }^{23,41,42}$ PWH develop a dysbiotic gut microbiota-that is, the gut microbiota displays alterations in the diversity, types, and relative proportions of bacteria that normally inhabit the GI tract. ${ }^{28}$ Furthermore, dysbiosis creates a vicious cycle with chronic inflammation promoting an environment abundant in pro-inflammatory species. ${ }^{24,28,43}$ Changes include a greater proportion of gram-negative bacterial family, Enterobacteriaceae, ${ }^{44}$ increased abundances of Prevotella particularly, Prevotella copri, and Erysipelotrichaceae, ${ }^{40}$ and depletion of Bifidobacterium and Bacteroides. ${ }^{24}$ Intriguingly, numerous taxa considered aerotolerant and generally part of 
the oral microbiota, not normally present in the gut, are elevated in PWH (Scott N. Peterson and Ronald J. Ellis, unpublished observation). Chronic oxidative stress in the gut may increase $\mathrm{O}_{2}$ levels in the gut enabling aerobic species to flourish in a normally strictly anaerobic environment.

The shift in gut microbiota to a Prevotella enriched composition is consistent with reports that a complex carbohydrate dominant diet also enriches for Prevotella. The extent to which Prevotella spp. versus P. copri, in particular, contribute to HIV-associated comorbidities remains unclear. The relative abundance of Prevotella is positively correlated with CXCL10/IP-10, a proinflammatory and antiangiogenic chemokine, levels and anti-correlated with $\mathrm{CD}^{+}{ }^{+} \mathrm{T}$ cells. ${ }^{45}$ Another study demonstrated that $P$. copri levels were positively associated with colonic $\mathrm{CD}^{+} \mathrm{c}^{+}$myeloid dendritic cells. ${ }^{46}$ Increased Prevotella has been implicated as a potential initiator to disturbance of GBA signaling. ${ }^{43}$ This change has significant consequences to cognitive function and neurological outcomes. The abundance of P. copri and Prevotella stercorea in PWH has been evaluated in numerous studies and found to be associated with sexual behavior rather than solely HIV status. ${ }^{47}$

A few studies examining HIV microbial dysbiosis have been conducted with a majority of men who have sex with men (MSM). In these studies, there has been an association with sexual orientation and an abundance of Prevotella enterotype in contrast to men who have sex with women who are rich in Bacteroides. ${ }^{48}$ However, this is independent of HIV status and more likely due to sexual behavior of anal intercourse and somewhat is ameliorated with ART initiation. ${ }^{49}$ Nevertheless, sexual behavior of anal intercourse has been shown to be a confounder in the link of HIV-related gut dysbiosis; studies involving microbiota in HIV should control for sex behavior with MSM status. ${ }^{50}$ This dysbiosis has been suggested to play a role in driving immune activation as opposed to previously suspected microbial translocation alone. ${ }^{51,52}$ However, a more recent study demonstrated that treatment with ART will have an effect on the microbiome that pervades sexual behavior but is correlated with. ${ }^{47}$

The impact of cannabis on gut microbiota has not been significantly reported in human populations to date or PWH. One study assessed sexual practice and drug use in $\mathrm{PWH}$, including cannabis on gut microbiota composition noting normalization of taxa commonly observed as dysbiotic in PWH. ${ }^{53}$ A decline in Prevotella abundance was associated with cannabis use ${ }^{53}$ (Table 1).
Another study examined the impact of cannabis on the gut microbiota and diet-induced obesity in mice. A limited number of taxa were quantified by quantitative polymerase chain reaction, and changes in some taxa were observed; however, interpretation of these results should be taken with caution as cannabis administration induced increased short-term hyperphagia. ${ }^{54}$ It is hypothesized that the effect of cannabis on gut microbiota is likely to occur in an indirect manner as the result of altered gut inflammation and homeostasis.

Gut bacteria control the differentiation and function of immune cells in the intestine, periphery, and brain. ${ }^{55-57}$ There is increasing evidence that gut microbiota and the immune system are critical factors in the pathogenesis of neurodevelopmental, psychiatric, and neurodegenerative disease as microbiota immunomodulation orchestrates communication between the gut and brain. ${ }^{58}$ Some of the cognitive domains are subject to immune-mediated central nervous system (CNS) injury from HIV induced microglial activation and contributing to HIV-related cognitive dysfunction. ${ }^{59}$ Furthermore, microglia is exquisitely responsive to the gut microbiome and commensal bacteria support the maintenance of microglia in normal homeostasis conditions. When microbiota is absent, microglia loses the ability to mature, becoming defected in differentiation, and function. ${ }^{55}$ In a study with germfree mice, severely-defected microglia led to impaired innate immune responses. Recolonization with a complex microbiota environment resulted in partial restoration of normal microglial features. ${ }^{55}$ LPS activates microglial cells leading to neuroinflammation and, when chronic, is a likely contributor to CNS pathologies, through a leaky gut-brain barrier (Fig. 2).

\section{The GBA}

The GBA encompasses bidirectional communication between the central and the enteric nervous systems (ENSs), linking emotional and cognitive centers of the brain with intestinal functions (Fig. 2) ${ }^{60}$ The autonomic nervous system, the ENS, and the hypothalamic pituitary adrenal axis mediate the communication of the GBA. Enteroendocrine signaling through enteroendocrine hormones (i.e., peptide YY [PYY], glucagonlike peptide-1 [GLP-1], and cholecystokinin, released into the serosa and systemic circulation) activates neuronal pathways, including extrinsic afferent neurons, sending messages to the CNS. These pathways mediate not only behaviors associated with food intake but also cognition and mood. Cannabis has been associated 
Table 1. Taxa Alterations by HIV and Cannabis

\begin{tabular}{|c|c|c|}
\hline \multicolumn{3}{|l|}{ Taxa altered by HIV infection } \\
\hline $\begin{array}{l}\text { Vujkovic-Cvijin et al. }{ }^{24} \text { [published } \\
\text { correction appears in Sci Transl } \\
\text { Med. } 2017 \text { Nov } 8 ; 9(415) \text { ] }\end{array}$ & $\begin{array}{l}\text { Proteobacteria } \uparrow \\
\text { Bacteroides } \downarrow\end{array}$ & $\begin{array}{l}\text { PWH displayed microbiota with increased potential for tryptophan catabolism } \\
\text { through kynurenine pathway. The HIV-infected dysbiotic composition correlated } \\
\text { with disease progression, higher levels of immune activation in the blood and } \\
\text { gut, and higher levels of tryptophan metabolism and inflammatory plasma } \\
\text { markers than in HIV uninfected. Greatest degrees of dysbiosis had elevated } \\
\text { markers of chronic inflammation and disease progression seen by high levels of } \\
\text { kynurenine production and IL-6. HIV altered microbiota has increase in potential } \\
\text { for kynurenine-tryptophan catabolism. Host-microbiome interaction with HIV } \\
\text { infection does not recover in ART effectiveness. A HIV-infected nonprogressor } \\
\text { participant displayed similar microbiota to that of uninfected participants. }\end{array}$ \\
\hline Dillon et al. ${ }^{28}$ & $\begin{array}{l}\text { Proteobacteria } \uparrow \\
\text { Firmicutes } \downarrow \\
\text { Prevotella } \uparrow \\
\text { Bacteroides } \downarrow\end{array}$ & $\begin{array}{l}\text { Colonic biopsies measured mucosal associated microbiota; Prevotella was } \\
\text { associated with T cell and mDC activation in the gut. Dysbiosis in untreated PWH is } \\
\text { dominated by an increased abundance of Prevotella and a proportional decrease } \\
\text { of anti-inflammatory bacteria that support epithelial barrier integrity with } \\
\text { untreated HIV. The proportional increase in Prevotella-Bacteroides ratio is } \\
\text { associated with increased mucosal DC and immune activation and a systemic } \\
\text { microbial translocation highlighting an existing relationship between the } \\
\text { microbiota community, intestinal inflammation, and systemic immune activation. }\end{array}$ \\
\hline Vázquez-Castellanos et al. ${ }^{105}$ & $\begin{array}{l}\text { Prevotella } \uparrow \\
\text { Succinivibrio } \uparrow \\
\text { Bacteroides } \downarrow \\
\text { Faecalibacterium } \downarrow\end{array}$ & $\begin{array}{l}\text { Metagenomic analysis of fecal samples revealed shifts in microbiota functional } \\
\text { distribution, including LPS, energy production, and translocation. Prevotella was } \\
7.8 \text { times higher in HIV infected than those without HIV. Interactions between the } \\
\text { microbiota and bacterial translocation could occur indirectly by cytokine } \\
\text { signaling. Abundance of Gram-negative bacteria in dysbiosis in PWH may } \\
\text { contribute to the burden of translocated abacterial antigens and immune } \\
\text { activation. }\end{array}$ \\
\hline Ling et al. ${ }^{40}$ & $\begin{array}{l}\text { Prevotella } \uparrow \\
\text { Megamonas } \uparrow \\
\text { Proteobacteria } \uparrow \\
\text { Faecalibacterium } \uparrow \\
\text { Bacteroides } \downarrow\end{array}$ & $\begin{array}{l}\text { In this Chinese cohort, examination of fecal samples The Firmicutes/Bacteroidetes } \\
\text { ratio remains high even with ART despite control of viral load by ART. Individuals } \\
\text { with HIV infection have dysbiosis, which remains unchanged but alters the } \\
\text { taxonomic composition with phylogenetic strains associated with inflammatory } \\
\text { cytokines. }\end{array}$ \\
\hline \multicolumn{3}{|l|}{ Taxa altered by cannabis } \\
\hline Fulcher et al. ${ }^{53}$ & $\begin{array}{l}\text { Ruminococcus } \uparrow \\
\text { Clostridium cluster IV } \uparrow \\
\text { Solobacterium } \uparrow \\
\text { Fusobacterium } \uparrow \\
\text { Acidaminococcus } \downarrow \\
\text { Prevotella } \downarrow \\
\text { Dialister } \downarrow \\
\text { Anaerostipes } \downarrow \\
\text { Dorea } \downarrow\end{array}$ & $\begin{array}{l}\text { Study examines sexual practice and drug use in PWH microbiome. } \\
\text { Cannabis was associated with abundance of Fusobacterium and Anaerotruncus and } \\
\text { a decrease in Prevotella, Acidaminococcus, Anaerostipes, and Dialister } \\
\text { abundances. } \\
\text { Methamphetamine and recent receptive anal intercourse use is associated with } \\
\text { increased pro-inflammatory taxa and cytokines. } \\
\text { Recent and frequency of receptive anal intercourse were associated with and } \\
\text { presence of gonorrhea and/or chlamydia, associated with dysbiosis. }\end{array}$ \\
\hline
\end{tabular}

ART, antiretroviral therapy; LPS, lipopolysaccharide; PWH, people living with HIV infection.

with significant increases in ghrelin and leptin, and decreases in PYY, consistent with the modulation of appetite hormones mediated through endocannabinoid receptors. ${ }^{61}$

\section{The Endocannabinoid System}

The endocannabinoid system encompasses a key interface between the gut microbiota, the immune system, and homeostasis of the human host. Two main endogenous cannabinoids, or endocannabinoids, are the brain-derived arachidonoyl ethanolamide, known as anandamide (AEA), and 2-arachidonoylglycerol (2AG) derived from lipid precursors, such as arachidonic acid, that are synthesized on demand. ${ }^{15,62}$ 2-AG has been isolated from both gut and brain tissue. ${ }^{62}$ Endocannabinoids in the postsynaptic neuron are released into the synaptic cleft and travel retrograde to the pre- synaptic neuron, where they inhibit neurotransmitter release; then they are rapidly metabolized by lipoxygenase, cyclooxygenase, and epoxygenases, enzymes involved in eicosanoid metabolism. AEA and 2-AG can also be degraded by hydrolysis into arachidonic acid and glycerol/ethanolamine by serine esterases. ${ }^{63}$

The cannabinoid receptors type-1 (CB1) and -2 (CB2) are located throughout the periphery and are concentrated in the GI tract. CB1 receptors are most abundant in the brain, where they function in neurotransmision. $^{15,16,62}$ The CB1 receptors are predominantly located in the nociceptive areas of the CNS, the cerebellum, hippocampus, limbic system, and the basal ganglia. These receptors have a limited concentration in the substantia nigra and periaqueductal gray matter, but are not observed in the medullary respiratory centers. CB1 receptors can also be expressed on immune, cardiac, and 




FIG. 2. Cannabis and the gut-brain axis. HIV infection leads to depletion of CD4 cells in the GALT, dysbiosis, and microbial translocation. These effects occur even in the context of successful antiretroviral therapy.

Endocannabinoids and Phytocannabinoids modulate gut inflammation and the microbiota stabilizing the blood-brain barrier pathway and reducing neuroinflammation.

testicular cells. ${ }^{64}$ In the GI tract, CB1 agonists are involved in feeding behavior, GI motility, satiety signaling, and energy balance. ${ }^{65} \mathrm{CB} 1$ peripheral activity includes lipogenesis and inhibition of adiponectin found at elevated levels in obese and diabetic individuals. CB1 signaling has been linked to increased levels of free fatty acids, low HDL, high triglycerides, and insulin resistance.

CB2 receptors are expressed mainly on membranes of immune and hematopoietic cells in the periphery. ${ }^{62}$ CB2 receptors are densely located in immune tissue and organs, expressed by diverse cell types, including macrophages, splenocytes, microglial, monocytes, and $\mathrm{T}$ cells resident in the thymus, spleen, and bone marrow and tonsils. ${ }^{65,66} \mathrm{CB} 2$ receptors located in the periphery have an immunomodulatory function playing an important role in pain reduction, inflammation, and physiological immune defense. ${ }^{64} \mathrm{CB} 2$ activation mediates a regulatory or suppressive anti-inflammatory effect but in some cases stimulation of CB2 stimulates tissue destruction and apoptosis, such as in cancer cells. ${ }^{67,68}$ Agonists of this receptor do not have psychoactive properties and are effective in mediating immunosuppression, preventing fibrosis or other organ scarring or in certain diseases triggering tissue damage. ${ }^{64}$

Antagonistic ligands for the endocannabinoid receptor (CB1) signaling in the gut have an anti-inflammatory effect with an attenuation of inflammatory cytokines, an increase in Akkermansia muciniphila, and decreases in Lachnospiraceae and Erysipelotrichaceae diversity in the gut. ${ }^{36}$ CB1 signaling is TLR4 dependent and known to be influenced by at least $A$. muciniphila. ${ }^{69}$ In one study, A. muciniphila administration increased the intestinal levels of endocannabinoids that control inflammation, the gut barrier, and gut peptide secretion. ${ }^{70}$ $\mathrm{CB} 1$ activation is anti-inflammatory in the gut. ${ }^{71}$

The dysbiotic gut environment and GALT are set up for an augmented cycle of inflammation and increased permeability of the gut epithelial barrier. The gut microbiota sends signals to the brain while affecting many functions through several pathways that comprise the GBA, including emotion and cognition (Fig. 2). HIV infection may, therefore, lead to the destabilization of the GBA through alterations of the immune system and gut, a possible consequence of ongoing gut epithelial barrier abnormalities and viral replication in the GALT persisting despite ART effectiveness in suppressing peripheral virus. ${ }^{39}$

HIV infection and associated gut inflammation disrupt the gut-endocannabinoid-brain interface, and these disturbances adversely affect brain function. Several lines of evidence suggest protective effects of cannabinoids on gut barrier function. While interactions between the gut microbiota and the endocannabinoid system are complex, there are likely to be opportunities to develop therapeutics targeting this axis. Binding of the plant-based cannabinoid THC to presynaptic cannabinoid receptors, in the CNS, principally CB1, mimics the biological properties of AEA, acting like a mood enhancer and stimulant of joy and happiness or euphoria. ${ }^{62}$ Cannabis administration is associated with significant increases in ghrelin and leptin and decreases in hormones that modulate appetite (e.g., PYY) mediated through endogenous cannabinoid receptors. ${ }^{61}$

\section{Phytocannabinoids}

One of the main cannabinoids, THC (Fig. 3a), was identified by Israeli physician, Dr. Raphael Mecholam, in the 1960s. ${ }^{14}$ THC is the principal psychoactive 

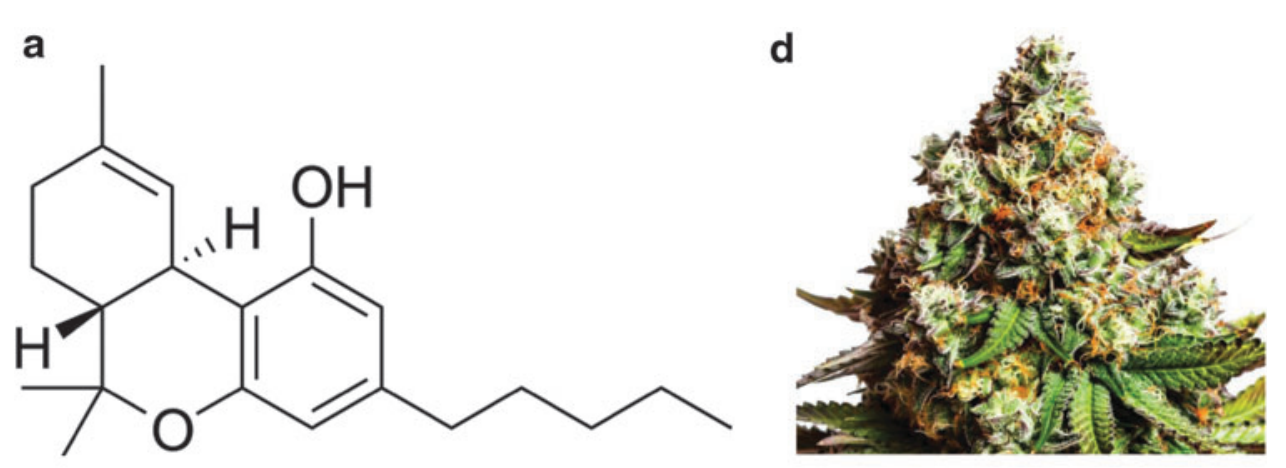

Cannabidiol (CBD)

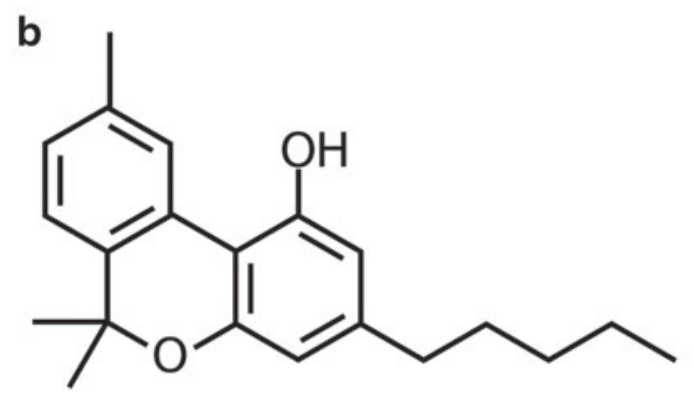

Cannabinol (CBN)



FIG. 3. Structure of (a) CBD, (b) CBN, (c) THC, (d) Capa Harley sativa-hybrid cultivar with indica-like effects due to high CBD component. A cultivar blend of sativa strains Harlequin and Green Crack with high CBD creating a balanced hybrid that acts like an indica dominant strain. Effect will alleviate pain and decrease stress yet creates a happy, ultra-relaxed feeling. Produces a cerebral euphoria as opposed to a high feeling with small amounts of inhalations. Smells earthy and woody (as described by Amsterdam Marijuana Seeds website).

component of cannabis. $\mathrm{CB} 1$ agonists such as terpenes and $\mathrm{CBD}$ in low doses can mitigate the psychoactive effects of THC. CBD (Fig. 3b) and CBN (Fig. 3c) act indirectly on $\mathrm{CB} 1$ and $\mathrm{CB} 2$ receptors. There are many more phytocannabinoid molecules that have an effect on the endocannabinoid system. Together they pro- duce an "entourage" or synergistic effect based on the ratios of these compounds that vary based on multiple factors, including plant strain and growing conditions. $^{72}$ This phenomenon may explain why isolated compounds derived from cannabis appear inert or only capable of exerting a limited effect. 
Medicinal and recreational cannabis comes in at least two species, Cannabis indica and Cannabis sativa, and in a variety of cultivars and formulations with different reported efficacies for treatment of various conditions. "Cultivar" is short for "cultivated variety" and represents not a taxonomic category, but a horticultural one, describing different plants that have been bred and selected by humans (Fig. 3d). ${ }^{73}$ Examples of cultivars include Acapulco Gold and Charlotte's Web. Acapulco Gold is a golden-leafed C. sativa strain originally from the Acapulco area of southwest Mexico. Charlotte's Web is a high-CBD, low-THC cannabis variety and extract marketed as a dietary supplement under federal law of the United States. More than 2,000 cultivars are available to consumers, but their chemical constituents and consistency have not been systematically characterized. This highlights the importance of providing consumers with consistent product information. CBD/THC extracts are reported to be more effective at treating pain compared to THC alone both in rodents and in human cancer-related pain. Species and cultivar differences in effects on the microbiome have not been studied.

There is also the chemovar referring to the different chemical varieties rather than the strain. ${ }^{74}$ Each of the chemovars will have variations in the cannabinoids isolated from the strain in various concentrations of $\mathrm{CBD}$, THC, cannabivarins, CBN, cannabigerol, and other chemicals such as terpenes. Each of them has specific functions and is able to elicit unique pharmacological actions and effects. ${ }^{74}$

Modes of administration are also important with respect to effect of cannabis and its therapeutic uses. Whether mode of administration differentially influences the gut microbiome is unknown. For example, one might expect orally administered cannabis to have more potent effects on the gut microbiome given high local concentrations than inhaled cannabis. But this has not been studied. Modes of administration will vary in time to onset of effects. Inhaled versions have a rapid onset of effect based on inhalations, whereas ingestion has a longer time to onset due to the transit to digestive processing and absorption. Finally, it is difficult to ascertain the dosing due to individual effects on physiology and tolerance.

The low systemic bioavailability of orally administered cannabinoids has led to exploration of other routes, including intranasal, transdermal, and transmucosal. These are possible because of the highly lipophilic nature of cannabinoids. Additional approaches to formulation that may influence bioavailability include salt formation (i.e., $\mathrm{pH}$ adjustment), co-solvency (e.g., ethanol, propylene glycol, PEG400, and so on), micellization (e.g., polysorbate 80, Cremophor ${ }^{\circledR}$ ELP, and so on), (nano)-(micro)-emulsification, complexation (e.g., cyclodextrins), and encapsulation in lipid-based formulations (e.g., liposomes) and nanoparticles. ${ }^{75-77}$

The U.S. Federal Drug Administration has approved three synthetic cannabinoids and one plant-derived cannabinoid. Marinol ${ }^{\circledR}$ (dronabinol) is approved for anorexia and nausea related to HIV and chemotherapy; Cesamet $^{\circledR}$ (nabilone) is approved to treat chemotherapyinduced nausea and chronic pain; and Epidiolex ${ }^{\circledR}$ is a plant-derived CBD indicated for the treatment of seizures associated with Lennox-Gastaut syndrome or Dravet syndrome in patients 2 years of age and older.

\section{Cannabinoids in the Brain and Gut}

Emerging findings suggest that cannabinoids can modulate the gut microbiota and inflammatory states by stabilizing blood-brain barrier function and reducing neuroinflammation. ${ }^{78}$ Neuroinflammation related to chronic immune activation, oxidative stress, and microbial translocation by a leaky gut barrier could affect the CNS through enteroendocrine signaling and the vagus nerve. ${ }^{79,80}$ These routes may interact with the better understood link between LPS translocation and chronic inflammation in the CNS due to microglial activation. ${ }^{81}$ Since inflammation and immune activation are believed to contribute to neurocognitive impairment in HIV, ${ }^{59}$ the antioxidant and anti-inflammatory properties and possible effects on gut barrier integrity of cannabinoids may favorably impact neurocognitive function. ${ }^{82}$

\section{Medicinal Phytocannabinoid Effects}

When THC and other exogenous cannabinoids interact with the endocannabinoid system, they can relieve pain as a result of neuromodulatory actions on both afferent pain signals and brain processing of pain. Several other potential therapeutic interventions have not been rigorously studied in randomized, controlled clinical trials. There is substantial evidence that THC stimulates appetite and reduces nausea. CBD is not psychoactive, acting as a serotonin 5-HT1A receptor agonist, and also has antioxidant and anti-inflammatory properties. ${ }^{83}$ In addition, although CBD has a low binding affinity for $\mathrm{CB} 1$ and $\mathrm{CB} 2$ receptors, it modulates several noncannabinoid receptors and ion channels and delays the "reuptake" of endogenous neurotransmitters such as anandamide and adenosine, by altering the binding of ligands to certain G-protein coupled receptors. 
Reports suggest that CBD may be effective for managing multiple anxiety based disorders, such as panic attacks, post-traumatic stress disorder, generalized anxiety, obsessive-compulsive disorders, and some cancers. ${ }^{84,85}$

Endocannabinoid signaling is known to influence gut barrier integrity, ${ }^{86}$ providing a highly relevant context for the study of the effects of cannabis. The endocannabinoid system in the large intestine interacts with the gut microbiota to regulate epithelial barrier permeability. ${ }^{87}$ The endogenous cannabinoid AEA, acting through $\mathrm{CB} 1$ and $\mathrm{CB} 2$ receptors, plays a pivotal role in maintaining immunological homeostasis and health in the gut. AEA contributes to the process by which the gut immune system actively tolerates microbial antigens. ${ }^{16}$ The bioactive lipid agonists and antagonists of cannabinoid receptors are known to have a direct effect on gut barrier function. Some CB1 and CB2 ligands are considered "gate openers," promoting inflammation due to increased permeability of food antigens and pathogen-associated molecular patterns (e.g., LPS). Other CB1 and CB2 ligands promote increased barrier function ("gatekeepers") and reduce inflammation. ${ }^{88}$ Enteroendocrine L cells are innervated by enteric glial cells and afferent neurons. ${ }^{89}$ Enteroendocrine L cells express endocannabinoid receptors (e.g., CB1 and CB2).

SIV infection was associated with gut epithelial barrier disruption, markers of increased inflammation/ immune activation (miR-150 downregulation and increased $\mathrm{T}$ cell activation), disrupting the translational control of IRAK1, and facilitating persistent GI inflammation. ${ }^{90}$ Previously published animal model studies support our focus on gut barrier permeability in the context of HIV and cannabis. One study demonstrated that chronic THC was associated with anti-inflammatory Th2 cytokine expression and reduced apoptosis among animals infected with SIV with markers of increased inflammation and immune activation in epithelial crypt cells. $^{90}$ These THC-mediated gut alterations were associated with reduced neuroinflammation measured as lower levels of TNF $\alpha$, IL1 $\beta$, IL6, and MCP1 in the striatum of SIV-infected rhesus macaques. ${ }^{91}$ These results may possibly translate to $\mathrm{PWH}$; however, there were notable sex-specific differences in THC outcomes in SIV infected macaques.

While THC mediated clinical differences in male rhesus macaques, reducing morbidity and mortality, as well as attenuation of SIV disease progression, female macaques did not demonstrate those protective benefits at similar doses. ${ }^{92,93}$ Male rhesus macaques had a reduction in plasma viral levels, decreased expression of tissue pro-inflammatory cytokines, and a decrease in intestinal apoptosis. ${ }^{92}$ Female macaques did not have protective benefits with alterations in SIV viral load and $\mathrm{CD} 4^{+} / \mathrm{CD}^{+}$ratio, with chronic daily $\mathrm{THC}$ administration. ${ }^{93}$ These contrasting effects may be due to endocrine hormonal differences, requiring more research to investigate the mechanisms for differences. Given these findings and the numerous studies reviewed and cohorts, the field of HIV-related gut dysbiosis is biased toward males and particularly MSM. Future studies should consider of purposively including adequate sampling of HIV-infected cis-females especially in examining the effects of phytocannabinoids.

Recently, studies have determined that cannabis is associated with reduced markers of immune activation and inflammation in CSF. This reduction was based on previous research demonstrating that selective stimulation of CB2 receptor leads to neuroinflammation and microglial activation. ${ }^{94-96}$ Thirty-six PWH and $21 \mathrm{HIV}$ negative participants underwent lumbar puncture and provided estimated days since their last cannabis use (median [range] 304 [1, > 1000]). More recent use of cannabis was associated with significantly lower CSF levels of IL-16 and C-reactive protein (CRP). These findings are consistent with the notion that CNS antiinflammatory effects of cannabinoids may be mediated directly through the microglial $\mathrm{CB} 2$ receptors or indirectly, for example, through cannabis-mediated alterations in gut microbiota composition, improved gut barrier function, or reduced translocation of proinflammatory bacterial products (Fig. 4).

\section{Clinical Endocannabinoid Deficiency Syndrome}

Over time, internal and external factors such as prolonged stress, environmental factors, poor nutrition, and overuse of cannabis may influence the ability to produce endocannabinoids. Clinical endocannabinoid deficiency syndrome (CEDS) has been linked to migraines, neuromuscular pain, and GI disorders. ${ }^{97}$ Specific symptoms and symptom clusters have been linked to a deficiency in the endocannabinoids, AEA and 2-AG. The symptom cluster with substantial evidence of CEDS is present for migraine, fibromyalgia, and irritable bowel syndrome. ${ }^{97}$ The endocannabinoid system regulates gut function, the CNS, and has a communicative relationship with the microbiome. Therefore, many other disorders and diseases are linked to a deficiency and dysfunction of the endocannabinoid system. ${ }^{97}$ Dysregulation of the endocannabinoids and CB2 receptors lead to many disorders affecting the liver, kidneys, CNS, 


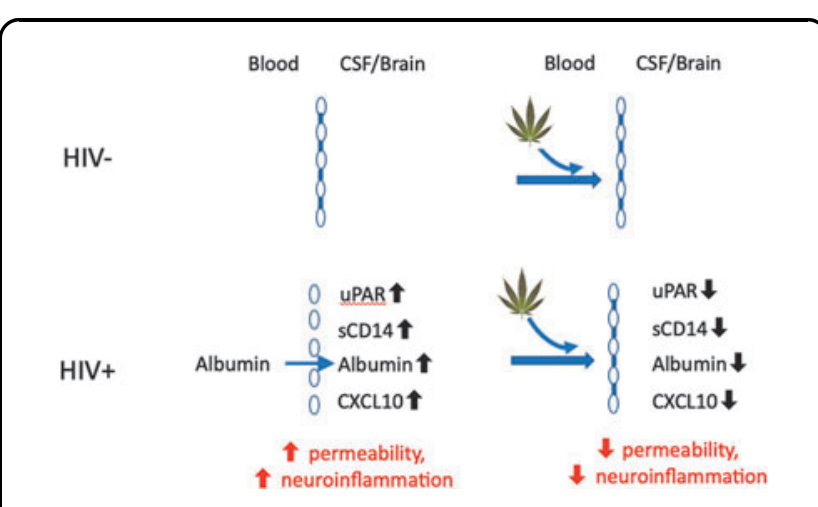

FIG. 4. Model of the blood-brain barrier before and after cannabis. HIV leads to increase in permeability and neuroinflammation through the increase in plasminogen activation system (UPAR), microbial translocation (SCD14), CXCL10/ IP10, and albumin levels across the blood-brain barrier. After administration of cannabis, there is a decrease in these markers, decreasing permeability and neuroinflammation.

neuromuscular, GI, immune system, lungs, bone, and mental health. ${ }^{62,98}$ Deficiency of endocannabinoids disrupts homeostasis. This provides an opportunity for the additional assessment of the therapeutic potential of phytocannabinoids, naturally-occurring cannabinoids in the cannabis, or hemp plants. These phytocannabinoids interact with the endocannabinoid system in the same way as endocannabinoids.

The GI microbiome recently was identified as a modulator of $\mathrm{BBB}$ integrity. There was a reduction in expression of the tight junction proteins occludin and claudin-5 on brain microvascular endothelial cells in germ-free mice (i.e., lacking a gut microbiota). Expression of these proteins and BBB integrity was restored after gut colonization with the butyrate producing species, Clostridium tyrobutyricum or by administration of butyrate. ${ }^{95}$

Probiotics improved gut integrity and enhanced endocannabinoid signaling. Zebrafish were treated with a probiotic formulation (VSL\#3; Lactobacillus spp., Bifidobacterium spp., and Streptococcus thermophilus) for 30 days. ${ }^{99}$ Compared to untreated animals, histological analysis of gut tissue from treated animals showed an intact epithelial barrier with increases in enterocyte length, villus length, and crypt depth. There was a reduction in epithelial and mesenchymal apoptotic cells, confirming molecular level changes of the pro-apoptotic factors casp3 (caspase 3 ) and BCL2 associated X (baxa), and an increase in antiapoptotic signals such as B cell lymphoma 2 (bcl2a). Probiotics also decreased the gene expression for fatty acid amide hydrolase (faah) and monoacylglycerol lipase $(m g l l)$, which are involved in the degradation of endocannabinoids AEA and 2-AG. One must take into consideration these metabolism pathways. Thus, probiotic treatment improved gut integrity and enhanced endocannabinoid signaling. ${ }^{99}$

\section{Discussion}

The influence of cannabis on host tissues, particularly gut permeability and its subsequent indirect effects on the gut microbiome, suggests significant potential therapeutic applications in HIV. Cannabis has been used for its medicinal properties for thousands of years in ancient cultures. ${ }^{100,101}$ Being a novice to cannabis use can be an intimidating issue for providers making recommendations to their patients. There are hundreds of strains that have names that are not based on structured nomenclature that clinicians are normally familiar with. ${ }^{102}$ Only recently, in the 1960 s have scientists begun to explore the properties of cannabis and even more recently the medicinal application in conjunction with Western medicine. Understanding the general physiological mechanism of endocannabinoids will support the framework in forming strategies to strain selection for symptom management.

Despite suppressive ART, PWH maintain a high symptom burden with GI disorders, HAND, depression/ anxiety, pain, and fatigue. In addition, $\mathrm{CD} 4{ }^{+} \mathrm{T}$ cell depletion and gut microbiota dysbiosis promote dysfunction of the gut epithelial barrier, resulting in a positive feedback loop sustained by increased microbial translocation of pro-inflammatory antigens such as LPS and subsequent immune activation and chronic inflammation. Consequences of these events in PWH are associated with poor health outcomes, including organ damage, cognitive decline, and decreased quality of life.

Phytocannabinoids may be a viable supplement to accommodate for deficiencies in the endocannabinoid system. Components of cannabis have an antiinflammatory and antioxidant effect addressing problems on a molecular and cellular level. Responsibly used, cannabis can be given as an antidepressant and for relief of post-traumatic stress disorder, sedative, and anticancer benefits and relief of obsessive behaviors. Benefits extend to symptomatic relief for symptoms like fatigue, poor appetite, depression, 
anxiety, insomnia, pain, nausea/vomiting, and cognitive changes. Clinicians will find confidence in educating themselves on the effects of cannabis to support a conversation with patients during office visits.

One caveat to widespread adoption by the medical community is that as of 2019, the Department of Justice Drug Enforcement Agency holds that cannabis is a controlled substance with no evidence of medical benefit and high potential of abuse, even with 33 of 50 states and the District of Columbia currently having state-legislated approval to dispense cannabis for medical purposes. ${ }^{103}$ Furthermore, the federal government enforces barriers and restrictions on studies investigating the benefits of cannabis due to federal restrictions. $^{104}$ Regardless of the discordance of laws between federal and state governments, patients are in fact using or interested in using cannabis to manage aspects of their health. Providers should have a working understanding of cannabis and its various effects on the body, including benefits and potential risks.

While effects of cannabis on gut barrier function have been studied in pre-clinical models, the translation to humans is uncertain. Evaluation of the gut microbiome in both PWH and HIV transgenic animals exposed to chronic cannabis is necessary to begin to test beneficial effects to correct gut permeability and dysbiosis. The additive effect of probiotics and cannabis may result in synergistic effects in terms of supporting healing of the gut and also the reduction of inflammation, immune activation, and neuropsychiatric disorders within the context of ART.

\section{Acknowledgments}

The authors acknowledge the Center for Medicinal Cannabis Research at the University of California, San Diego for supporting this work.

\section{Author Disclosure Statement}

No competing financial interests exist.

\section{Funding Information}

Natalie L. Wilson has received support from T32 NR007081, R25MH083620, and 3R24AI067039-14W1. Ronald J. Ellis is funded, in part, by National Institutes of Health R01 DA043430. All authors have no conflicts to disclose.

\section{References}

1. Whiting PF, Wolff RF, Deshpande S, et al. Cannabinoids for medical use: a systematic review and meta-analysis. JAMA. 2015;313:2456-2473.
2. Abrams DI, Jay CA, Shade SB, et al. Cannabis in painful HIV-associated sensory neuropathy: a randomized placebo-controlled trial. Neurology. 2007;68:515-521.

3. Zurier RB, Burstein SH. Cannabinoids, inflammation, and fibrosis. FASEB J. 2016;30:3682-3689.

4. Lutge EE, Gray A, Siegfried N. The medical use of cannabis for reducing morbidity and mortality in patients with HIV/AIDS. Cochrane Database Syst Rev. 2013:CD005175.

5. Ellis RJ, Toperoff W, Vaida F, et al. Smoked medicinal cannabis for neuropathic pain in HIV: a randomized, crossover clinical trial. Neuropsychopharmacology. 2009;34:672-680.

6. Abrams DI, Hilton JF, Leiser RJ, et al. Short-term effects of cannabinoids in patients with HIV-1 infection: a randomized, placebo-controlled clinical trial. Ann Intern Med. 2003;139:258-266.

7. Prospero-Garcia O, Amancio-Belmont O, Becerril Melendez AL, et al. Endocannabinoids and sleep. Neurosci Biobehav Rev. 2016;71:671-679.

8. Andreae $\mathrm{MH}$, Carter GM, Shaparin N, et al. Inhaled cannabis for chronic neuropathic pain: a meta-analysis of individual patient data. J Pain. 2015; 16:1221-1232.

9. Wilson NL, Azuero A, Vance DE, et al. Identifying symptom patterns in people living with HIV disease. J Assoc Nurses AIDS Care. 2016;27:121132.

10. Brooks E, Gundersen DC, Flynn E, et al. The clinical implications of legalizing marijuana: are physician and non-physician providers prepared? Addict Behav. 2017;72:1-7.

11. Carlini BH, Garrett SB, Carter GT. Medicinal cannabis: A survey among health care providers in Washington State. Am J Hosp Palliat Care. 2017; 34:85-91.

12. Azofeifa A, Mattson ME, Schauer G, et al. National estimates of marijuana use and related indicators-National Survey on Drug Use and Health, United States, 2002-2014. MMWR Surveill Summ. 2016;65:1-28.

13. Bonn-Miller MO, Oser ML, Bucossi MM, et al. Cannabis use and HIV antiretroviral therapy adherence and HIV-related symptoms. J Behav Med. 2014;37:1-10.

14. Di Marzo V. Cannabinoids. John Wiley \& Sons, Inc.: Chichester, West Sussex, United Kingdom, 2014.

15. Pacher $P, B A ́ T$ kai $S$, Kunos $G$. The endocannabinoid system as an emerging target of pharmacotherapy. Pharmacol Rev. 2006;58: 389-462.

16. Acharya N, Penukonda S, Shcheglova T, et al. Endocannabinoid system acts as a regulator of immune homeostasis in the gut. Proc Natl Acad Sci U S A. 2017;114:5005-5010.

17. Li C, Jones PM, Persaud SJ. Role of the endocannabinoid system in food intake, energy homeostasis and regulation of the endocrine pancreas. Pharmacol Ther. 2011;129:307-320.

18. Manuzak JA, Gott TM, Kirkwood JS, et al. Heavy cannabis use associated with reduction in activated and inflammatory immune cell frequencies in antiretroviral therapy-treated human immunodeficiency virusinfected individuals. Clin Infect Dis. 2018;66:1872-1882.

19. Chaillon A, Nakazawa M, Anderson C, et al. Effect of cannabis use on human immunodeficiency virus DNA during suppressive antiretroviral therapy. Clin Infect Dis. 2020;70:140-143.

20. Thames AD, Mahmood Z, Burggren AC, et al. Combined effects of HIV and marijuana use on neurocognitive functioning and immune status. AIDS Care. 2016;28:628-632.

21. Milloy MJ, Marshall B, Kerr T, et al. High-intensity cannabis use associated with lower plasma human immunodeficiency virus-1 RNA viral load among recently infected people who use injection drugs. Drug Alcohol Rev. 2015;34:135-140.

22. Ellis RJ, Peterson SN, Li Y, et al. Recent cannabis use in HIV is associated with reduced inflammatory markers in CSF and blood. Neurol Neuroimmunol Neuroinflamm. 2020;7:e809.

23. Somsouk M, Estes JD, Deleage C, et al. Gut epithelial barrier and systemic inflammation during chronic HIV infection. AIDS. 2015;29:43-51.

24. Vujkovic-Cvijin I, Dunham RM, Iwai S, et al. Dysbiosis of the gut microbiota is associated with HIV disease progression and tryptophan catabolism. Sci Transl Med. 2013;5:193ra191.

25. Brenchley JM, Douek DC. The mucosal barrier and immune activation in HIV pathogenesis. Curr Opin HIV AIDS. 2008;3:356-361.

26. Klatt NR, Funderburg NT, Brenchley JM. Microbial translocation, immune activation, and HIV disease. Trends Microbiol. 2013;21:6-13. 
27. Brenchley JM, Price DA, Schacker TW, et al. Microbial translocation is a cause of systemic immune activation in chronic HIV infection. Nat Med. 2006;12:1365-1371.

28. Dillon SM, Lee EJ, Kotter CV, et al. An altered intestinal mucosal microbiome in HIV-1 infection is associated with mucosal and systemic immune activation and endotoxemia. Mucosal Immunol. 2014;7:983-994.

29. Dillon SM, Kibbie J, Lee EJ, et al. Low abundance of colonic butyrateproducing bacteria in HIV infection is associated with microbial translocation and immune activation. AIDS. 2017;31:511-521.

30. Jenabian MA, El-Far M, Vyboh K, et al. Immunosuppressive tryptophan catabolism and gut mucosal dysfunction following early HIV infection. J Infect Dis. 2015;212:355-366.

31. Sandler NG, Douek DC. Microbial translocation in HIV infection: causes, consequences and treatment opportunities. Nat Rev Microbiol. 2012;10: 655-666.

32. McHardy IH, Li X, Tong M, et al. HIV Infection is associated with compositional and functional shifts in the rectal mucosal microbiota. Microbiome. 2013;1:26.

33. Dillon SM, Frank DN, Wilson CC. The gut microbiome and HIV-1 pathogenesis: a two-way street. AIDS. 2016;30:2737-2751.

34. Jandhyala SM, Talukdar R, Subramanyam C, et al. Role of the normal gut microbiota. World J Gastroenterol. 2015;21:8787-8803.

35. Chow J, Tang H, Mazmanian SK. Pathobionts of the gastrointestinal microbiota and inflammatory disease. Curr Opin Immunol. 2011;23: 473-480.

36. Mehrpouya-Bahrami $P$, Chitrala KN, Ganewatta MS, et al. Blockade of CB1 cannabinoid receptor alters gut microbiota and attenuates inflammation and diet-induced obesity. Sci Rep. 2017;7:15645.

37. d'Ettorre G, Rossi G, Scagnolari C, et al. Probiotic supplementation promotes a reduction in T-cell activation, an increase in Th17 frequencies, and a recovery of intestinal epithelium integrity and mitochondrial morphology in ART-treated HIV-1-positive patients. Immun Inflamm Dis. 2017;5:244-260.

38. Brenchley JM, Schacker TW, Ruff LE, et al. CD4 + T cell depletion during all stages of HIV disease occurs predominantly in the gastrointestinal tract. J Exp Med. 2004;200:749-759.

39. Thompson CG, Gay CL, Kashuba ADM. HIV persistence in gut-associated lymphoid tissues: Pharmacological challenges and opportunities. AIDS Res Hum Retroviruses. 2017;33:513-523.

40. Ling $Z$, Jin $C$, Xie T, et al. Alterations in the fecal microbiota of patients with HIV-1 infection: an observational study in a Chinese population. Sci Rep. 2016;6:30673.

41. Mudd JC, Brenchley JM. Gut mucosal barrier dysfunction, microbial dysbiosis, and their role in HIV-1 disease progression. J Infect Dis. 2016; 214 Suppl 2:S58-S66.

42. Zevin AS, McKinnon L, Burgener A, et al. Microbial translocation and microbiome dysbiosis in HIV-associated immune activation. Curr Opin HIV AIDS. 2016;11:182-190.

43. Lozupone CA, Li M, Campbell TB, et al. Alterations in the gut microbiota associated with HIV-1 infection. Cell Host Microbe. 2013;14: 329-339.

44. Ellis CL, Ma ZM, Mann SK, et al. Molecular characterization of stool microbiota in HIV-infected subjects by panbacterial and order-level $16 \mathrm{~S}$ ribosomal DNA (rDNA) quantification and correlations with immune activation. J Acquir Immune Defic Syndr. 2011;57:363-370.

45. Kaur US, Shet A, Rajnala N, et al. High Abundance of genus Prevotella in the gut of perinatally HIV-infected children is associated with IP-10 levels despite therapy. Sci Rep. 2018;8:17679.

46. Dillon SM, Lee EJ, Kotter CV, et al. Gut dendritic cell activation links an altered colonic microbiome to mucosal and systemic T-cell activation in untreated HIV-1 infection. Mucosal Immunol. 2016;9:24-37.

47. Vujkovic-Cvijin I, Sortino O, Verheij E, et al. HIV-associated gut dysbiosis is independent of sexual practice and correlates with noncommunicable diseases. Nat Commun. 2020;11:2448.

48. Noguera-Julian M, Rocafort M, Guillen $Y$, et al. Gut microbiota linked to sexual preference and HIV infection. EBioMedicine. 2016;5:135-146.

49. Armstrong AJS, Shaffer M, Nusbacher NM, et al. An exploration of Prevotella-rich microbiomes in HIV and men who have sex with men. Microbiome. 2018;6:198.

50. Tuddenham S, Koay WL, Sears C. HIV, Sexual orientation, and gut microbiome interactions. Dig Dis Sci. 2020;65:800-817.
51. Neff $C P$, Krueger $\mathrm{O}$, Xiong $\mathrm{K}$, et al. Fecal microbiota composition drives immune activation in HIV-infected individuals. EBioMedicine. 2018;30: 192-202.

52. Li SX, Sen S, Schneider JM, et al. Gut microbiota from high-risk men who have sex with men drive immune activation in gnotobiotic mice and in vitro HIV infection. PLoS Pathog. 2019;15:e1007611.

53. Fulcher JA, Hussain SK, Cook R, et al. Effects of substance use and sex practices on the intestinal microbiome during HIV-1 infection. J Infect Dis. 2018;218:1560-1570.

54. Cluny NL, Keenan CM, Reimer RA, et al. Prevention of diet-induced obesity effects on body weight and gut microbiota in mice treated chronically with delta9-tetrahydrocannabinol. PLoS One. 2015;10: e0144270.

55. Erny D, Hrabe de Angelis AL, Jaitin D, et al. Host microbiota constantly control maturation and function of microglia in the CNS. Nat Neurosci. 2015;18:965-977.

56. Matcovitch-Natan O, Winter DR, Giladi A, et al. Microglia development follows a stepwise program to regulate brain homeostasis. Science. 2016;353:aad8670.

57. Rooks MG, Garrett WS. Gut microbiota, metabolites and host immunity. Nat Rev Immunol. 2016;16:341-352.

58. Fung TC, Olson CA, Hsiao EY. Interactions between the microbiota, immune and nervous systems in health and disease. Nat Neurosci. 2017;20: $145-155$.

59. Rubin LH, Sacktor N, Creighton J, et al. Microglial activation is inversely associated with cognition in individuals living with HIV on effective antiretroviral therapy. AIDS. 2018;32:1661-1667.

60. Mayer EA, Tillisch K, Gupta A. Gut/brain axis and the microbiota. J Clin Invest. 2015;125:926-938.

61. Riggs PK, Vaida F, Rossi SS, et al. A pilot study of the effects of cannabis on appetite hormones in HIV-infected adult men. Brain Res. 2012;1431: 46-52.

62. Begg $M$, Pacher $P$, Bátkai $S$, et al. Evidence for novel cannabinoid receptors. Pharmacol Ther. 2005;106:133-145.

63. Turcotte C, Chouinard F, Lefebvre JS, et al. Regulation of inflammation by cannabinoids, the endocannabinoids 2-arachidonoyl-glycerol and arachidonoyl-ethanolamide, and their metabolites. J Leukoc Biol. 2015; 97:1049-1070.

64. Pacher $P$, Mechoulam R. Is lipid signaling through cannabinoid 2 receptors part of a protective system? Prog Lipid Res. 2011;50:193-211.

65. McPartland JM, Duncan M, Di Marzo V, et al. Are cannabidiol and Delta(9) -tetrahydrocannabivarin negative modulators of the endocannabinoid system? A systematic review. Br J Pharmacol. 2015;172: 737-753.

66. Munro S, Thomas KL, Abu-Shaar M. Molecular characterization of a peripheral receptor for cannabinoids. Nature. 1993;365:61-65.

67. McKallip RJ, Lombard C, Fisher M, et al. Targeting CB2 cannabinoid receptors as a novel therapy to treat malignant lymphoblastic disease. Blood. 2002;100:627-634.

68. Lombard C, Nagarkatti M, Nagarkatti P. CB2 cannabinoid receptor agonist, JWH-015, triggers apoptosis in immune cells: potential role for CB2-selective ligands as immunosuppressive agents. Clin Immunol. 2007;122:259-270.

69. Plovier $\mathrm{H}$, Everard A, Druart $\mathrm{C}$, et al. A purified membrane protein from Akkermansia muciniphila or the pasteurized bacterium improves metabolism in obese and diabetic mice. Nat Med. 2017;23:107-113.

70. Everard A, Belzer C, Geurts L, et al. Cross-talk between Akkermansia muciniphila and intestinal epithelium controls diet-induced obesity. Proc Natl Acad Sci U S A. 2013;110:9066-9071.

71. Villanueva-Millan MJ, Perez-Matute P, Recio-Fernandez E, et al. Differential effects of antiretrovirals on microbial translocation and gut microbiota composition of HIV-infected patients. J Int AIDS Soc. 2017;20: 21526.

72. Russo EB. The case for the entourage effect and conventional breeding of clinical cannabis: no "strain," no gain. Front Plant Sci. 2018;9:1969.

73. Hazekamp A, Fischedick JT. Cannabis-from cultivar to chemovar. Drug Test Anal. 2012;4:660-667.

74. Bruni N, Della Pepa C, Oliaro-Bosso S, et al. Cannabinoid delivery systems for pain and inflammation treatment. Molecules. 2018;23:2478.

75. Lawrence MJ, Rees GD. Microemulsion-based media as novel drug delivery systems. Adv Drug Deliv Rev. 2000;45:89-121. 
76. Kumari A, Yadav SK, Yadav SC. Biodegradable polymeric nanoparticles based drug delivery systems. Colloids Surf B Biointerfaces. 2010;75:1-18.

77. Allen TM, Cullis PR. Liposomal drug delivery systems: from concept to clinical applications. Adv Drug Deliv Rev. 2013;65:36-48.

78. Josue Perez-Santiago SG, Ajay Bharti, Debralee Cookson, Robert K. Heaton, et al. The human gut microbiome and HIV-associated neurocognitive disorders. Paper presented at: CROI 2017; Seattle, WA.

79. Dockray GJ. Enteroendocrine cell signalling via the vagus nerve. Curr Opin Pharmacol. 2013;13:954-958.

80. Hong S, Banks WA. Role of the immune system in HIV-associated neuroinflammation and neurocognitive implications. Brain Behav Immun. 2015;45:1-12.

81. Bussi C, Peralta Ramos JM, Arroyo DS, et al. Autophagy down regulates pro-inflammatory mediators in BV2 microglial cells and rescues both LPS and alpha-synuclein induced neuronal cell death. Sci Rep. 2017;7:43153.

82. Petrosino $S$, Verde $R$, Vaia $M$, et al. Anti-inflammatory properties of cannabidiol, a nonpsychotropic cannabinoid, in experimental allergic contact dermatitis. J Pharmacol Exp Ther. 2018;365:652-663.

83. De Filippis D, Steardo A, D'Amico A, et al. Differential cannabinoid receptor expression during reactive gliosis: a possible implication for a nonpsychotropic neuroprotection. ScientificWorldJournal. 2009;9:229-235.

84. Blessing EM, Steenkamp MM, Manzanares J, et al. Cannabidiol as a potential treatment for anxiety disorders. Neurotherapeutics. 2015;12:825-836.

85. Daris B, Tancer Verboten M, Knez Z, et al. Cannabinoids in cancer treatment: therapeutic potential and legislation. Bosn J Basic Med Sci. 2019;19:14-23.

86. Cani PD. Crosstalk between the gut microbiota and the endocannabinoid system: impact on the gut barrier function and the adipose tissue. Clin Microbiol Infect. 2012;18 Suppl 4:50-53.

87. Muccioli GG, Naslain D, Backhed F, et al. The endocannabinoid system links gut microbiota to adipogenesis. Mol Syst Biol. 2010;6:392.

88. Cani PD, Plovier H, Van Hul M, et al. Endocannabinoids-at the crossroads between the gut microbiota and host metabolism. Nat Rev Endocrinol. 2016;12:133-143.

89. Yoo BB, Mazmanian SK. The enteric network: interactions between the immune and nervous systems of the gut. Immunity. 2017;46:910926.

90. Kumar V, Torben W, Kenway CS, et al. Longitudinal examination of the intestinal lamina propria cellular compartment of simian immunodeficiency virus-infected rhesus macaques provides broader and deeper insights into the link between aberrant MicroRNA expression and persistent immune activation. J Virol. 2016;90:5003-5019.

91. Simon L, Song K, Vande Stouwe C, et al. Delta9-Tetrahydrocannabinol (Delta9-THC) promotes neuroimmune-modulatory MicroRNA profile in striatum of simian immunodeficiency virus (SIV)-infected macaques. J Neuroimmune Pharmacol. 2016;11:192-213.

92. Molina PE, Amedee AM, LeCapitaine NJ, et al. Modulation of gut-specific mechanisms by chronic delta(9)-tetrahydrocannabinol administration in male rhesus macaques infected with simian immunodeficiency virus: a systems biology analysis. AIDS Res Hum Retroviruses. 2014;30:567-578.

93. Amedee AM, Nichols WA, LeCapitaine NJ, et al. Chronic Delta(9)tetrahydrocannabinol administration may not attenuate simian immunodeficiency virus disease progression in female rhesus macaques. AIDS Res Hum Retroviruses. 2014;30:1216-1225.

94. Ashton JC, Glass M. The cannabinoid CB2 receptor as a target for inflammation-dependent neurodegeneration. Curr Neuropharmacol. 2007;5:73-80.

95. Braniste V, Al-Asmakh M, Kowal C, et al. The gut microbiota influences blood-brain barrier permeability in mice. Sci Transl Med. 2014;6: 263 ra158.

96. Lin $L$, Yihao $T$, Zhou $F$, et al. Inflammatory regulation by driving microglial M2 polarization: neuroprotective effects of cannabinoid receptor-2 activation in intracerebral hemorrhage. Front Immunol. 2017;8:112.
97. Russo EB. Clinical endocannabinoid deficiency reconsidered: current research supports the theory in migraine, fibromyalgia, irritable bowel, and other treatment-resistant syndromes. Cannabis Cannabinoid Res. 2016;1:154-165.

98. Kogan NM, Mechoulam R. Cannabinoids in health and disease. Dialogues Clin Neurosci. 2007;9:413-430.

99. Gioacchini G, Rossi G, Carnevali O. Host-probiotic interaction: new insight into the role of the endocannabinoid system by in vivo and ex vivo approaches. Sci Rep. 2017;7:1261.

100. Brand EJ, Zhao Z. Cannabis in Chinese medicine: are some traditional indications referenced in ancient literature related to cannabinoids? Front Pharmacol. 2017;8:108.

101. Russo EB. Cannabis and epilepsy: an ancient treatment returns to the fore. Epilepsy Behav. 2017;70:292-297.

102. Abood ME, Pertwee RG. Cannabinoids. Springer: Berlin; New York, 2005.

103. ProCon.org. 30 Legal Medical Marijuana States and DC. Retrieved from http://medicalmarijuana.procon.org/view.resource.php?resourcelD= 000881 Accessed September 3, 2019.

104. National Academies of Sciences, Engineering, and Medicine. The health effects of cannabis and cannabinoids: the current state of evidence and recommendations for research. The National Academies Press: Washington, DC, 2017.

105. Vázquez-Castellanos JF, Serrano-Villar S, Latorre A, et al. Altered metabolism of gut microbiota contributes to chronic immune activation in HIV-infected individuals. Mucosal Immunol. 2015;8:760-772.

Cite this article as: Wilson NL, Peterson SN, Ellis RJ (2021) Cannabis and the gut-brain axis communication in HIV infection, Cannabis and Cannabinoid Research 6:2, 92-104, DOI: 10.1089/can.2020.0037. 